\title{
An Exploratory Study on the Feasibility of a Foot Gear Type Energy Harvester Using a Textile Coil Inductor
}

\author{
Hyun-Seung Cho*, Jin-Hee Yang*, Seon-Hyung Park**, Kwang-Seok Yun***, \\ Yong-Jun Kim ${ }^{\S}$ and Joo-Hyeon Lee ${ }^{\dagger}$
}

\begin{abstract}
This research developed a textile coil inductor, in which conductive yarn was wound spirally onto textile, and produced an energy harvesting module utilizing a cylindrical compression coil spring structure to allow a permanent magnet to spin in the center hole of the coil inductor. The study confirmed through a pilot test that the voltage increased as the number of laminated layers of the coil inductor increased. Five subjects were tested in the energy harvesting measuring experiment after producing a sports shoe insole-mounted energy harvesting module. While the subjects executed sports motions such as walking and running at five given frequencies, the peak-to-peak voltage was measured by an oscilloscope and the accumulated energy voltage of the calculated rms voltage (Vrms) and the peak power $(\mu \mathrm{W})$ were derived. The output voltage increased as the frequency increased and the average $\mathrm{Vp}-\mathrm{p}(\mathrm{V})$ of the five subjects was $0.53 \mathrm{~V}$, the average peak power $(\mu \mathrm{W})$ was $0.289 \mu \mathrm{W}$, and the Vrms (V) was $0.065 \mathrm{~V}$. This research is significant in that it suggests the possibility of an energy harvesting module based upon the textile coil inductor emerging from the former shoes' energy generator packaging method for heavy shoe types by developing a lightweight, flexible, and humanfriendly footgear module structure.
\end{abstract}

Keywords: Conductive thread, Textile coil inductor, Magnetic-induced energy, Energy harvesting module, Sport shoe design

\section{Introduction}

As the standard of living has recently elevated and shoes with many functions and designs have hit stores based on the demands of customers with a variety of needs, the purpose of shoes is now not only to protect our feet, but they have also become a device which is equipped with many functions. For example, the production has increased for safety shoes for laborers with special tasks, diet shoes without heels, and shoes for diabetic patients. These are designed for comfort and performance.

Meanwhile, as the environmental pollution problems from the use of fossil fuels all over the world are on the rise, there are studies in progress on alternative energy such as wind energy, hydraulic energy, solar energy, and bio-energy. This type of energy is clean, ever-renewable and never on the brink of being exhausted. On the other hand, these types of energy have a few of disadvantages in terms of efficiency and economic feasibility because they

$\dagger$ Corresponding Author: Dept. of Clothing \& Textiles, Yonsei University, Korea. (ljhyeon@yonsei.ac.kr)

* Institute of Symbiotic Life-TECH, Yonsei University, Korea. (hyunseung-cho@yonsei.ac.kr)

** Dept. of Fashion Design, Joongbu University, Korea. (pshyung@joongbu.ac.kr)

*** Dept. of Electronic Engineering, Sogang University, Korea. (ksyun@sogang.ac.kr)

$\S$ School of Mechanical Engineering, Yonsei University, Korea. (yjk@yonsei.ac.kr)

Received: October 21, 2015; Accepted: March 29, 2016 lack practicality in sites where relatively high energy is needed due to too low energy density; additionally, solar power and wind power can easily become affected by the climate, thereby requiring an auxiliary generator. For this reason, alternative energy is required and a series of studies on 'Green Energy Harvesting' are in progress to overcome the crisis. The green energy harvesting technique is associated with an energy supplying device, which converts diverse energy sources such as vibration, heat and force into electronic power. The device is semi-permanent and does not require any maintenance expenses. Therefore, the development of green energy harvesting techniques which change diverse forms of energy in the surrounding environment into electronic energy has been expanding as an effort to promote a productive system which converts various physical energy forms in the human body into practical and meaningful power.

According to the 'Organic and Printed Electronics Association', it is predicted that the energy harvesting clothing market will be rising soon after the coming of the IT-integrated smart clothing market around the year 2020 [1]. There are many efforts towards converting the diverse forms of energy that are generated in the human body to electronic power that can be used for mobile devices, as well as efforts towards a new alternative energy source in line with an independent energy generating plan [2]. There are studies on low power supply technology such as energy harvesting devices; these are aimed at product groups that 
consume low power for portable or wearable electronic devices $[3,4]$.

The University of Southampton in the United Kingdom reported that piezoelectric harvesting film producing technology, which is used to convert walking and running energy into electronic energy in footwear, will be completed around the year 2015 [5]. In 2011, Zegna Sport in Italy commercialized the Ecotech Solar Jacket, in which the latest technology and solar power technology are combined and the researchers from Wisconsin University successfully developed a shoe-mounted battery charging system, which is able to harvest about $20 \mathrm{~W}$ through walking motions [6]. Likewise, the development of various IT-integrated functional sportswear products is on the rise with the increase in consumer demand. The demand for power supply will increase as well based on the growth rate of ITintegrated clothing products with the growth of the energy harvesting clothing market as an energy supplying system.

Recently, as a result of the energy harvesting research, there have been many trials in developing an independent energy generating system that converts kinetic energy to electronic energy. For example, by converting the kinetic energy to electronic energy, the energy of an independent power supply shoe should be sufficient enough for it to be used in our daily lives. The independent power supply, however, has to be durable to bear the repeated external force from walking due to the extra purchase of the independent power supply device. It also has to have a reasonable production cost to rationalize the production process. The traditional shoe with the current independent power supply technology has problems in that it is inconvenient for customers due to the irritating feeling from the low elasticity and durability of the materials; also, it can possibly become damaged by the repeated force from the wearer.

The purpose of this study is to develop a new footwear design for the lightweight power harvesting module that provides comfortable and wearable user satisfaction and cannot become damaged by repeated pressure; this will be done by developing a flexible independent power supplying device based on the law of electromagnetic induction.

\section{Principles of Electromagnetic Energy Harvesting}

The basic principle on which almost all electromagnetic generators are based is Faraday's law of electromagnetic induction [8]. The principle of Faraday's law is that the voltage, or electromotive force (emf), induced in a circuit is proportional to the time rate of change of the magnetic flux linkage of that circuit, i. e.

$$
V=-\frac{\mathrm{d} \phi}{\mathrm{d} t}
$$

where $V$ is the generated voltage or induced emf and $\phi$ is the flux linkage. In most generator implementations, the circuit consists of a coil of wire of multiple turns and the magnetic field is created by permanent magnets. In this case, the voltage induced in an $N$ turn coil is given by:

$$
V=-\frac{\mathrm{d} \Phi}{\mathrm{d} t}=-N \frac{\mathrm{d} \phi}{\mathrm{d} t}
$$

where $\Phi$ is the total flux linkage of the $N$ turn coil and can be approximated as, $N \phi$, and in this case $\phi$ can be interpreted as the average flux linkage per turn. In general, the flux linkage for a multiple turn coil should be evaluated as the sum of the linkages for the individual turns, i.e.

$$
\Phi=\sum_{i=1}^{N} \int_{A i} B \cdot \mathrm{d} A
$$

where $B$ is the magnetic field flux density over the area of the $i$ th turn. In the case where the flux density can be considered uniform over the area of the coil, the integral can be reduced to the product of the coil area, number of turns and the component of flux density normal to the coil area, $\Phi=N B A \sin (\alpha)$, where $\alpha$ is the angle between the coil area and the flux density direction. Consequently, in such a case, the induced voltage is given by:

$$
V=-N A \frac{\mathrm{d} B}{\mathrm{~d} t} \sin (\alpha)
$$

\section{Materials and Methods}

In this study, we developed an energy harvesting module with a flexible textile coil inductor and tested the possibility of a lightweight, flexible, body-friendly energy harvesting device after mounting it in a sports shoe insole.

\subsection{The development of the textile coil inductor}

The main material of the coil inductor was a metalpolyester hybrid conductive yarn; it was composed of one strand of 75 denier polyester, two strands of $50 \mu \mathrm{m}$ thick
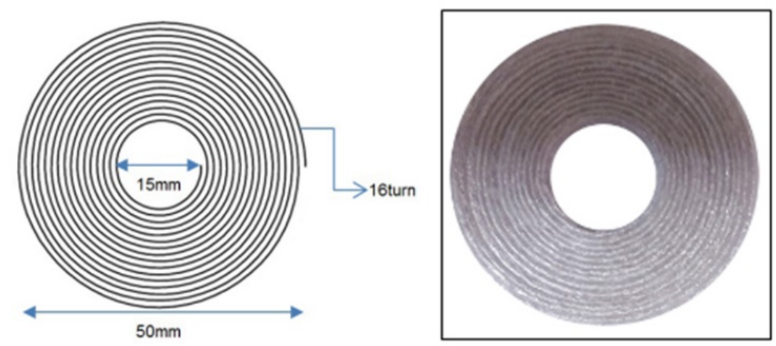

Fig. 1. Single layer of the textile coil inductor 

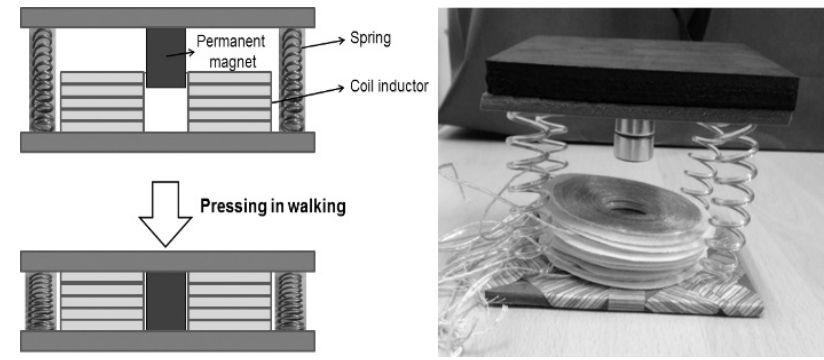

Fig. 2. Conceptual diagram of the magnetic-induced energy-harvesting principle in walking using a multi-layered textile coil inductor

metal yarn (sliver plated nickel thread) and six strands of $30 \mu \mathrm{m}$ thick metal yarn. This processed nine strands into 19-plys conductive yarn with $0.123 \Omega / \mathrm{m}$ in resistance level. The surface of the conductive yarn was coated by PVC and wound spirally on the polyester textile, applying air-core coils form to swirl form. The size of the coil inductor was $50 \mathrm{~mm}$ in diameter and the center hole was $15 \mathrm{~mm}$ in diameter; the coil had 16 turns (Fig. 1).

\subsection{Production of the basic energy harvesting module mock-up}

The energy harvesting module from the textile coil inductor is a device that uses electromagnetic induction to generate electronic power. Electromagnetic induction is the production of an electromotive force across a conductor when it is exposed to a varying magnetic field [7].

In order to generate the induced current, as the magnetic field on the coil changed, the coil inductor was multilayered with five layers. These layers were placed in a coil spring structure and designed for a $12 \mathrm{~mm}$ permanent magnet (neodymium) to become interlinked in the center hole of the coil inductor due to walking (Fig. 2).

The energy harvesting module in Fig. 2 was designed for a permanent magnet to oscillate and generate an induced current through a magnetic field change on the air core coil. According to this principle, voltage was generated by the applied pressure from repeated walking by a wearer. Therefore, sports shoes that contain its structure can provide a simple, lightweight, and flexible module structure compared to traditional shoes.

\subsection{Pilot test}

In order to verify the energy harvesting effect from the energy harvesting module of the multi-layered structure, the output voltage was measured by an oscillator (Signal Force GW-V4/PA30E, Data Physics Corporation). The voltage increase was measured at each number of laminated coil inductor layers within $4 \mathrm{~Hz}$ in oscillation frequency (Fig. 3). As a result, the voltage increased linearly with the number of laminated layers and $30.65 \mathrm{mV}$ (Vp-p) was measured at the 5th layer (Table 1, Fig. 4).
Table 1. Voltage at each number of laminated coil inductor layers (1 5 layers; oscillation frequency: $4 \mathrm{~Hz}$ )

\begin{tabular}{c|c|c}
\hline $\begin{array}{c}\text { The number of coil } \\
\text { inductor layers }\end{array}$ & $\begin{array}{c}\text { The number of coil } \\
\text { inductor turns }\end{array}$ & $\mathrm{Vp}-\mathrm{p}(\mathrm{mV})$ \\
\hline 1 & 16 & 4.65 \\
\hline 2 & 32 & 11.25 \\
\hline 3 & 48 & 19.05 \\
\hline 4 & 64 & 25.85 \\
\hline 5 & 80 & 30.65 \\
\hline
\end{tabular}

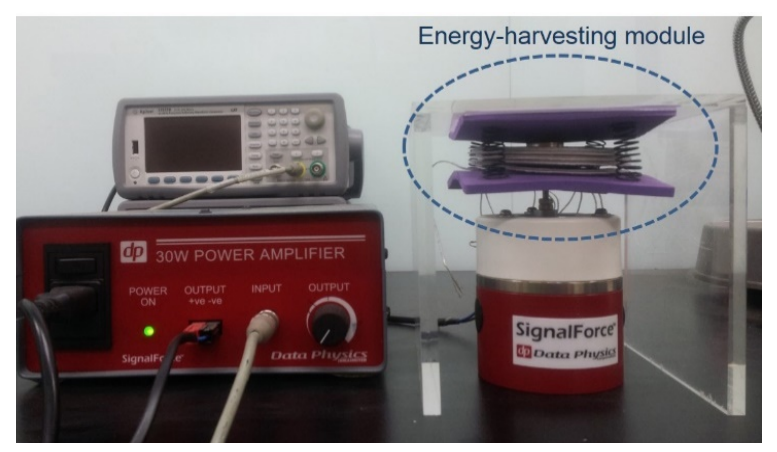

Fig. 3. Measuring experiment on voltage by an oscillator

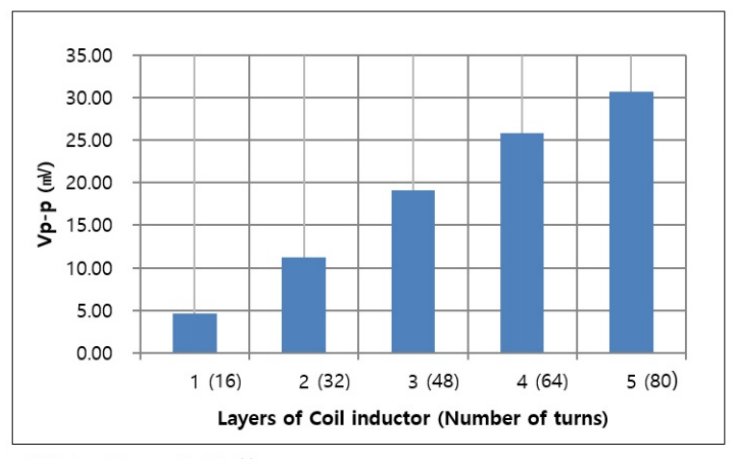

* Noise Vp-p : $3.15 \mathrm{mV}$

Fig. 4. Peak-to-peak voltage (Vp-p) increase at each number of laminated coil inductor layers

\subsection{The main experiment}

As a result of the pilot test, the voltage increase was verified with the laminated layers and the optimal height of the number of layers was 10 units $(3 \mathrm{~cm})$; the wearers did not experience an irritating feeling from the sports shoe insole. Hence, the basic mock-up of the energy harvesting module was revised to a 10-layer structure (Fig. 5) and five male subjects in their twenties were selected (Table 2). They wore sports shoes in which the multi-layered energy harvesting module was mounted, and walked (to the right) as in Fig. 6. They walked at five different frequencies within $0.5 \sim 2.5 \mathrm{~Hz}(0.5 \mathrm{~Hz}$ : slow walking at $2.56 \mathrm{~km} / \mathrm{hr}$; $1 \mathrm{~Hz}$ : normal walking at $5.12 \mathrm{~km} / \mathrm{hr} ; 1.5 \mathrm{~Hz}$ : fast walking at $6.8 \mathrm{~km} / \mathrm{hr} ; 2 \mathrm{~Hz}$ : normal running at $10.24 \mathrm{~km} / \mathrm{hr}$; $2.5 \mathrm{~Hz}$ : fast running at $12.8 \mathrm{~km} / \mathrm{hr}$ ). The outputs were recorded by an oscilloscope. In other words, the peak-topeak voltage $(\mathrm{Vp}-\mathrm{p})$, peak power $(\mu \mathrm{W})$, and Vrms $(\mathrm{V})$ were 
Table 2. Body types of the subjects (somatotype)

\begin{tabular}{c|c|c|c|c}
\hline Subject & Sex & Age & Height $(\mathrm{cm})$ & Weight $(\mathrm{kg})$ \\
\hline 1 & Male & 26 & 179 & 77 \\
\hline 2 & Male & 27 & 175 & 75 \\
\hline 3 & Male & 26 & 181 & 78 \\
\hline 4 & Male & 26 & 174 & 60 \\
\hline 5 & Male & 25 & 175 & 70 \\
\hline
\end{tabular}
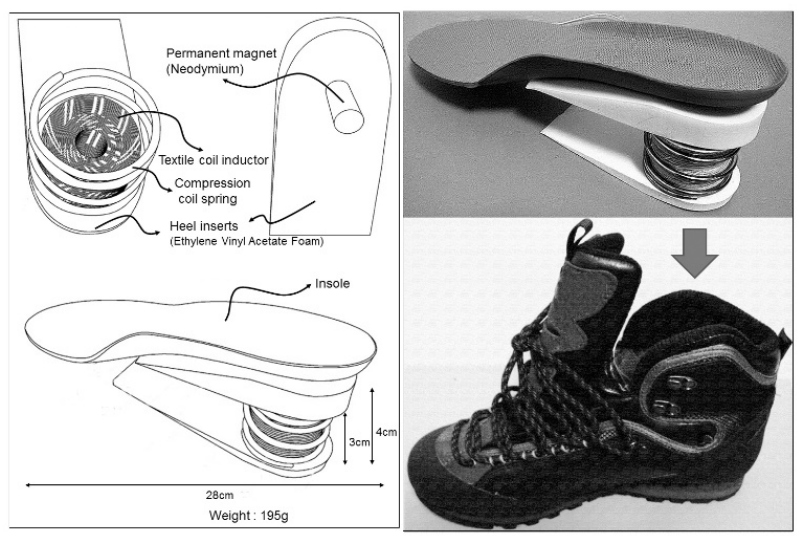

Fig. 5. Sports shoe with the mounted magnetic-induced energy harvesting module

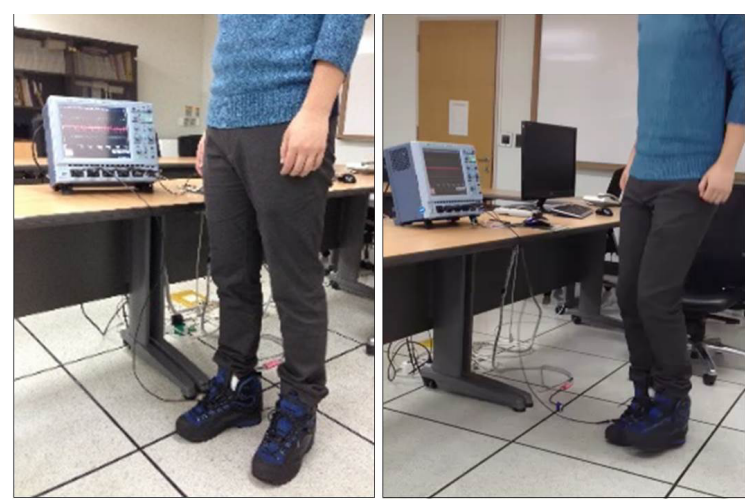

Fig. 6. Measuring experiment on the output voltage from walking and running

measured to calculate the accumulated voltage energy by an oscilloscope after having the subjects exercise (walking and running) for 20 seconds at each given frequency within $0.5 \sim 2.5 \mathrm{~Hz}$.

\section{Results and Discussion}

As the frequency increased within 20 seconds, the voltage followed the same pattern from $0.5 \sim 2.5 \mathrm{~Hz}$ and the average peak-to-peak voltage (Vp-p) of the five subjects was $0.53 \mathrm{~V}$ at $2.5 \mathrm{~Hz}$; in addition, the individual test results of the subjects showed the same, however, depending on the subject, at $2.5 \mathrm{~Hz}$, the highest Vp-p was $0.73 \mathrm{~V}$, and the lowest was $0.42 \mathrm{~V}$ (Table 3, Fig. 7). It was assumed that the difference in walking pattern, strength, and body shape
Table 3. Peak-to-peak voltage (Vp-p) at each frequency for walking and running

\begin{tabular}{c|c|c|c}
\hline \multirow{2}{*}{ Frequency (Hz) } & \multicolumn{3}{|c}{ Vp-p (V) } \\
\cline { 2 - 4 } & Mean & Maximum & Minimum \\
\hline 0.5 & 0.17 & 0.20 & 0.14 \\
\hline 1.0 & 0.26 & 0.33 & 0.21 \\
\hline 1.5 & 0.37 & 0.52 & 0.30 \\
\hline 2.0 & 0.43 & 0.57 & 0.33 \\
\hline 2.5 & 0.53 & 0.73 & 0.42 \\
\hline * Coil Resistance $: 4.8$ & Ohm; $\mathrm{R}_{\mathrm{L}}=1$ MOhm (oscilloscope)
\end{tabular}

* Coil Resistance : 4.8 Ohm ; $\mathrm{R}_{\mathrm{L}}=1 \mathrm{MOhm}$ (oscilloscope)

Table 4. Peak power $(\mu W)$ at each frequency for walking and running

\begin{tabular}{c|c|c|c}
\hline \multirow{2}{*}{ Frequency $(\mathrm{Hz})$} & \multicolumn{3}{|c}{ Peak power $(\mu \mathrm{N})$} \\
\cline { 2 - 4 } & Mean & Maximum & Minimum \\
\hline 0.5 & 0.030 & 0.040 & 0.020 \\
\hline 1.0 & 0.072 & 0.109 & 0.044 \\
\hline 1.5 & 0.142 & 0.270 & 0.090 \\
\hline 2.0 & 0.195 & 0.325 & 0.109 \\
\hline 2.5 & 0.289 & 0.533 & 0.176 \\
\hline * Coil Resistance $: 4.8$ & $\mathrm{Ohm} ; \mathrm{R}_{\mathrm{L}}=1$ MOhm (oscilloscope)
\end{tabular}

* Coil Resistance : $4.8 \mathrm{Ohm} ; \mathrm{R}_{\mathrm{L}}=1 \mathrm{MOhm}$ (oscilloscope)

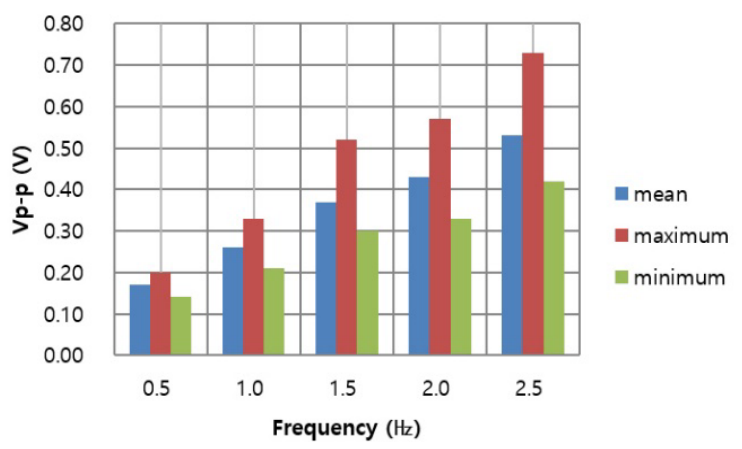

Fig. 7. Peak-to-peak voltage (Vp-p) increase at each frequency for walking and running

among the subjects affected the voltage level.

The result of the peak power $(\mu \mathrm{N})$ within 20 seconds showed the highest value at $2.5 \mathrm{~Hz}$. The average was 0.289 \lrcorner $\mathrm{N}$ and therefore double the amount of energy harvesting in the actual sports activities was predicted for when both feet are actually used. Depending on the subject, the highest peak power $(\mu \mathrm{W})$ was $0.533 \mu \mathrm{W}$, and the lowest was 0.176 $\iota \mathrm{W}$, showing a relatively big difference (Table 4, Fig. 8).

Furthermore, in 20 seconds, the Vrms (V) exhibited a continual increase from $0.5 \mathrm{~Hz}$ to $2.5 \mathrm{~Hz}$, and had the highest voltage at $2.5 \mathrm{~Hz}$ for all of them. The average Vrms (V) was $0.065 \mathrm{~V}$, the highest Vrms (V) was $0.070 \mathrm{~V}$, and the lowest was $0.056 \mathrm{~V}$ (Table 5, Fig. 9).

The above results are for the voltage gained from only one shoe (i.e., the right shoe) with the energy harvesting module within 20 seconds (the energy harvesting module was mounted in the right shoe in this study).

According to the test results, the estimated energy harvesting values from both sports shoes were measured. From the measuring process of the voltage conveyed to a 1 $\mathrm{M} \Omega$ load, the Vrms (V) at $2.5 \mathrm{~Hz}$ was $0.065 \mathrm{~V}$, the voltage 
Table 5. Vrms (V) at each frequency for walking and running

\begin{tabular}{c|c|c|c}
\hline \multirow{2}{*}{ Frequency (Hz) } & \multicolumn{3}{|c}{ Vrms (V) } \\
\cline { 2 - 4 } & Mean & Maximum & Minimum \\
\hline 0.5 & 0.021 & 0.030 & 0.017 \\
\hline 1.0 & 0.029 & 0.035 & 0.020 \\
\hline 1.5 & 0.045 & 0.056 & 0.035 \\
\hline 2.0 & 0.056 & 0.064 & 0.048 \\
\hline 2.5 & 0.065 & 0.070 & 0.056 \\
\hline
\end{tabular}

* Coil Resistance : $4.8 \mathrm{Ohm} ; \mathrm{R}_{\mathrm{L}}=1 \mathrm{MOhm}$ (oscilloscope)

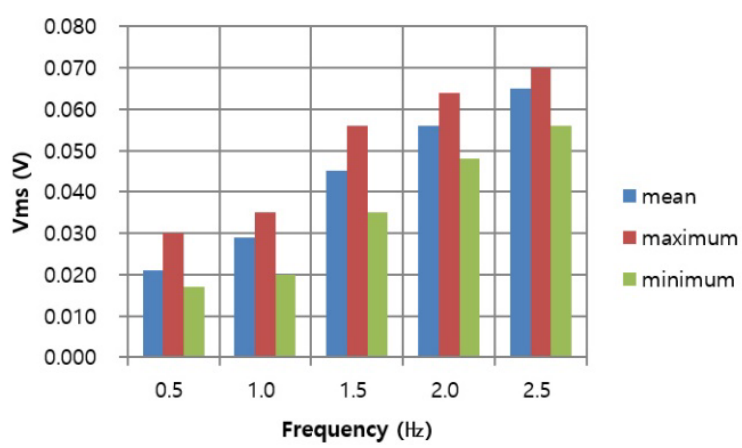

Fig. 9. Vrms (V) increase at each frequency for walking and running

was $4.23 \mathrm{nW}\left(\operatorname{Prms}=\mathrm{V}^{2} \mathrm{rms} / \mathrm{R}\right)$ and the energy harvesting value was $5.076 \mu J$ from both sports shoes for 10 minutes of exercise.

Assuming the actual exercise environment is used, the energy harvesting value from both sides of the sports shoes was $12.0 \mu \mathrm{J}$ when the distance for one step for a male subject while walking was $0.8 \mathrm{~m}$ at $8 \mathrm{~km} / \mathrm{hr}$ (fast walking) for 50 minutes. That is to say that $8 \mathrm{~km} / \mathrm{hr}$ was converted to $1.5 \mathrm{~Hz}$ in frequency from walking. Then, the measured Vrms (V) was $0.045 \mathrm{~V}$ and the Prms was $2 \mathrm{nW}$. Likewise, more energy harvesting was expected from both sports shoes after active motions such as walking and running for a longer period of time.

\section{Conclusion}

This study wound the conductive yarn spirally on the polyester textile, developed the textile coil inductor and produced a sports shoe energy harvesting module by designing a permanent magnet that oscillates in the center hole of a coil inductor with a multi-layered cylindrical compression coil spring structure. The peak-to-peak voltage, peak power $(\mu \mathrm{W})$ and $\operatorname{Vrms}(\mathrm{V})$ were measured and the accumulated voltage was obtained for five subjects executing sports motions (walking and running) by an oscilloscope at each frequency from 0.5 to $2.5 \mathrm{~Hz}$ in 20 seconds. As the frequency increased, the voltage increased as well; the average values of the Vp-p (V), peak power $(\mu \mathrm{W})$ and Vrms $(\mathrm{V})$ of the five subjects were $0.53 \mathrm{~V}, 0.289$ $\mu \mathrm{W}$ and $0.065 \mathrm{~V}$ at $2.5 \mathrm{~Hz}$.
Based on this study, the harvested voltage value from the developed sports shoe itself is relatively low. However, more energy harvesting will be expected through on-going development of the textile coil inductor. For instance, by placing the conductive yarn coil on the front and back side it is possible to double the number of laminated layers. Additionally, the conductive ink can be printed on the textile, and by applying the multi-layering method with the inductor, which has a larger number of turns than the former, the voltage will increase.

This study is significant in that it suggests the possibility of an energy-harvesting module based upon the textile coil inductor emerging from the former shoes' energy generator packaging method for heavy shoe types by developing a lightweight, flexible, and human-friendly footgear module structure.

\section{Acknowledgements}

This research was supported by the Pioneer Research Center Program through the National Research Foundation of Korea funded by the Ministry of Science, ICT \& Future Planning (2010-0019313).

\section{References}

[1] Klaus Hecker, "Organic and Printed ElectronicsEnabling Electronics Everywhere", Wearable Technologies Conference 2013, Munich, ICM, February $4^{\text {th }}, 2013$.

[2] Boram Yang and Kwang-Seok Yun, "Efficient energy harvesting from human motion using wearable piezoelectric shell structures", Solid-State Sensors, Actuators and Microsystems Conference (TRANSDUCERS), pp. 2646-2649, $16^{\text {th }}$ International, Beijing 5-9 June 2011.

[3] R. J. M. Vullers, R. van Schaijk, I. Doms, C. Van Hoof and R. Mertens, "Micropower energy harvesting”, Solid-State Electronics, 53, pp. 684-693, 2009.

[4] P. D. Mitcheson, E. M. Yeatman, , G. K. Rao, A. S. Holmes, and T. C. Green, "Energy harvesting from human and machine motion for wireless electronic devices", Proceedings of the IEEE, 96(9), pp. 14571486, 2008.

[5] Sciencedaily, "Clothing to power personal computers", Retrieved from http://www.sciencedaily.com/releases/ 2010/08/100817143810.htm.

[6] Techno-Science.net, Recharger ses appareils mobiles en marchant. Retrieved from http://www.technoscience.net/?onglet=news\&news $=9512,2011$.

[7] kunjak Park, Electromagnetics (Easy to learn), bookshill, Seoul, 2013, pp. 174-175.

[8] S. Priya and D. J. Inman, Energy Harvesting Technologies, Springer, 2009, pp. 130-132. 


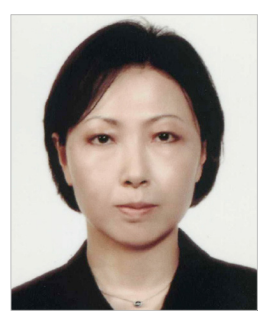

Joo-Hyeon Lee Joo-Hyeon Lee received her Ph.D. degree in clothing \& textiles from Yonsei University. Her research interests are smart clothing, sensors, energy harvesting, and wearable system.

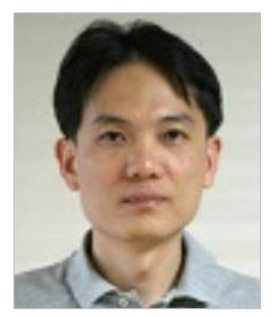

Kwang-Seok Yun Kwang-Seok Yun received his Ph.D. degrees in Electronics Engineering from Korea Advanced Institute of Science and Technology. His current research area includes micro integrated systems, MEMS, and micro sensors and actuators.

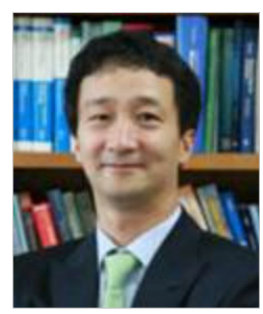

Yong-Jun Kim Yong-Jun Kim received his Ph.D. degree in electrical engineering from Georgia Institute of Technology. His current research includes general micromachining, bio and environmental sensors, and flexible electronic packaging.

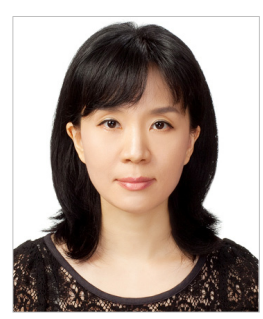

Hyun-Seung Cho Hyun-Seung Cho received her $\mathrm{Ph} . \mathrm{D}$. degree in cognitive science from Yonsei University. Her research interests are smart clothing, energy harvesting, and wearable system.

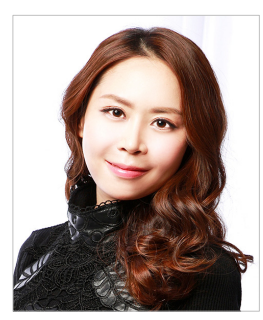

Jin-Hee Yang Jin-Hee Yang received her Ph.D. degree in clothing \& textiles from Yonsei University. Her research interests are smart clothing, energy harvesting, and wearable system.

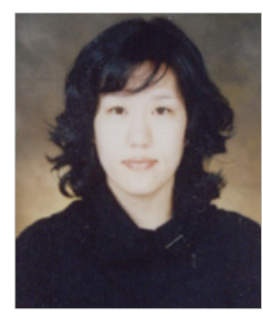

Seon-Hyung Park Seon-Hyung Park received her $\mathrm{Ph}$.D. degree in clothing \& textiles from Yonsei University. Her research interests are smart clothing, energy harvesting, and wearable system. 